\title{
Severe COVID-19 initially presenting as mesenteric adenopathy
}

\author{
Sakura Noda ${ }^{1,2}$ (D) Jimmy $\mathrm{Ma}^{3} \cdot$ Erin K. Romberg ${ }^{1,2} \cdot$ Rafael E. Hernandez ${ }^{4,5} \cdot$ Mark R. Ferguson $^{1,2}$
}

Received: 20 May 2020 / Revised: 1 July 2020 / Accepted: 27 July 2020 / Published online: 10 October 2020

(C) Springer-Verlag GmbH Germany, part of Springer Nature 2020

\begin{abstract}
Coronavirus disease 2019 (COVID-19) can present with abdominal pain in children and adults. Most imaging findings have been limited to characteristic lung findings, as well as one report of bowel-ischemia-related findings in adults. We report a case of COVID-19 in a healthy teenager who initially presented with isolated mesenteric adenopathy, typically a self-limited illness, which progressed to severe illness requiring intensive care before complete recovery. The boy tested negative for COVID-19 twice by polymerase chain reaction (PCR) from upper respiratory swabs before sputum PCR resulted positive. A high index of suspicion should be maintained for COVID-19 given the continued emergence of new manifestations of the disease.
\end{abstract}

Keywords Abdomen · Adolescent · Computed tomography · Coronavirus · COVID-19 · Lymph nodes · Mesentery · Severe acute respiratory syndrome coronavirus 2 (SARS-CoV-2)

\section{Introduction}

Severe acute respiratory syndrome coronavirus 2 (SARSCoV-2), the causative agent of coronavirus disease 2019 (COVID-19), caused almost 13 million confirmed infections globally as of July 14, 2020 [1]. While most infected individuals are asymptomatic or experience only self-limited respiratory illness, others develop severe multisystemic illness resulting in more than 570,000 deaths worldwide [1]. Because of its highly contagious and potentially lethal nature, identifying the varied presentations of COVID-19 is important for prompt and accurate diagnosis.

We describe a pediatric patient who presented with abdominal pain and was diagnosed with mesenteric adenopathy -

Sakura Noda

sakura.noda@seattlechildrens.org

1 Department of Radiology, Seattle Children's Hospital, M/S MA.7.220, P.O. Box 5731, Seattle, WA 98104-5005, USA

2 Department of Radiology, University of Washington, Seattle, WA, USA

3 Division of Infectious Diseases, Department of Medicine, University of Washington, Seattle, WA, USA

4 Division of Infectious Diseases, Seattle Children's Hospital, Seattle, WA, USA

5 Department of Pediatrics, University of Washington, Seattle, WA, USA typically a self-limited illness - but who was ultimately diagnosed with COVID-19. Notably, no initial clinical symptoms or CT findings suggested COVID-19 respiratory infection.

Gastrointestinal symptoms including diarrhea, vomiting and abdominal pain are known manifestations of COVID19. Reported imaging findings in patients with gastrointestinal symptoms have mostly been limited to lung-base abnormalities [2]. One recent study of adults with COVID-19 identified abdominal imaging findings of bowel wall thickening, pneumatosis, and portal venous gas suggestive of ischemia secondary to small-vessel thrombosis, some confirmed by pathology [3]. Isolated mesenteric adenopathy has not been described as a manifestation of COVID-19.

\section{Case report}

A generally healthy, immunized, non-obese White 17-yearold boy presented to an outside emergency department (ED) with 3 days of initially moderate progressing to severe abdominal pain focused in the right lower quadrant, fever as high as $103^{\circ} \mathrm{F}$, and vomiting without diarrhea. He had seen a friend with an upper respiratory infection 2 weeks prior but had no known COVID-19 exposure. The boy's medical history included a Staphylococcus aureus abscess and cellulitis requiring incision and drainage without history of unusual, recurrent or difficult-to-treat infections. 
At the outside hospital ED, the boy's white blood cell count, mononucleosis screen, and nasopharyngeal SARSCoV-2 polymerase chain reaction (PCR) testing was unrevealing. Abdominopelvic $\mathrm{CT}$ with intravenous contrast agent demonstrated a normal appendix, diffuse mesenteric lymphadenopathy, mesenteric fat stranding and mild splenomegaly (Fig. 1). There were no other lymphadenopathy, gross vascular thrombus or vascular irregularity, or pulmonary findings to suggest COVID-19. He was discharged home with instructions for supportive care and close primary care follow-up with the diagnosis of mesenteric adenopathy.

The boy presented to our pediatric ED 2 days later because of worsening abdominal pain and persistent vomiting. In the interim he had also developed a productive cough and severe headache. Physical examination demonstrated pallor, tachycardia and diffusely tender abdomen with right lower quadrant guarding. Pulse oximetry was $100 \%$ on room air, the boy was not tachypneic, chest retractions were not present, and the lungs were clear to auscultation. No skin rashes were noted. Because of high clinical suspicion for acute appendicitis, we repeated ultrasound exam. The visualized portions of the appendix appeared normal, although the tip was obscured. Because of the boy's worsening clinical status after fluid resuscitation with at least $4 \mathrm{~L}$ of intravenous normal saline, we repeated abdominopelvic $\mathrm{CT}$. Abdominal findings were similar to prior findings, but the lung bases demonstrated new peripheral basilar ground-glass and solid consolidation as well as interlobular septal thickening. There were minimal right pleural effusion and trace ascites, but no gallbladder wall thickening or significant bowel wall thickening.

In the ED, he developed hypoxemia requiring nonrebreather mask and was admitted to the pediatric intensive care unit (PICU). Repeat mid-turbinate nasal swab SARS$\mathrm{CoV}-2$ testing was negative. Because of worsening hypoxic respiratory failure and the suspicious lung $\mathrm{CT}$ findings, we performed a repeat SARS-CoV-2 PCR on expectorated sputum, and this test was positive. Extensive nasopharyngeal, serum and stool testing for other infectious agents was negative (Table 1). Blood and urine cultures were negative. Elevated erythrocyte sedimentation rate $(42 \mathrm{~mm} / \mathrm{h}$, normal 0 $15 \mathrm{~mm} / \mathrm{h})$, C-reactive protein $(42.4 \mathrm{mg} / \mathrm{dL}$, normal $\leq 0.8 \mathrm{mg} /$ $\mathrm{dL})$, IL-6 (36.5 pg/mL, normal 0-9 pg/mL), D-dimer (2.2 $\mathrm{mcg} / \mathrm{mL}$, normal $\leq 0.5 \mathrm{mcg} / \mathrm{mL})$, ferritin $(593 \mu \mathrm{g} / \mathrm{L}$, normal $10-300 \mu \mathrm{g} / \mathrm{L})$, white blood cell count $\left(15.6 \mathrm{~K} / \mathrm{mm}^{3}\right.$, normal $\left.4.5-11 \mathrm{~K} / \mathrm{mm}^{3}\right)$, absolute neutrophil count $\left(13,728 \mathrm{~mm}^{3}\right)$, and neutrophil-to-lymphocyte ratio of 14.9 were consistent with hyperinflammatory response. The patient also exhibited slightly elevated B-type natriuretic peptide (BNP, $565 \mathrm{pg} /$ $\mathrm{mL}$, normal $0-41 \mathrm{pg} / \mathrm{mL}$ ) but normal cardiac function and no pericardial effusion by echocardiogram. Renal and liver function tests were normal.

In the PICU, the boy required supportive care including noninvasive positive pressure support (continuous and bilevel positive airway pressure) and diuretics. Respiratory and abdominal symptoms improved after 1 day. Vancomycin, ceftriaxone and metronidazole, initiated in the ED out of concern for sepsis, were stopped after 2 days when SARS-CoV-2 was positive. The boy was transferred to the medical floor on the third day after admission. Remdesivir, an investigational antiviral drug, was administered on the third day after admission but discontinued because of the boy's rising alanine aminotransferase and improving condition. The boy was discharged home on the sixth day after admission feeling well and near clinical baseline, with residual fatigue and cough.

\section{Discussion}

Isolated mesenteric adenopathy has not been reported as a presentation of COVID-19. Although we did not obtain tissue sampling to prove that the mesenteric adenopathy was secondary to COVID-19, the boy eventually developed chest CT findings and severe hyperinflammatory response consistent with COVID-19, tested positive for SARS-CoV-2 by PCR from sputum, and recovered with primarily supportive care. Alternative causes of abdominal pain such as appendicitis and
Fig. 1 Initial CT. a, b Axial image (a) and coronal reformat (b) of abdominopelvic CT with intravenous contrast agent in a 17-year-old boy demonstrate enlarged lymph nodes (straight arrows) and adjacent fat stranding (curved arrows)
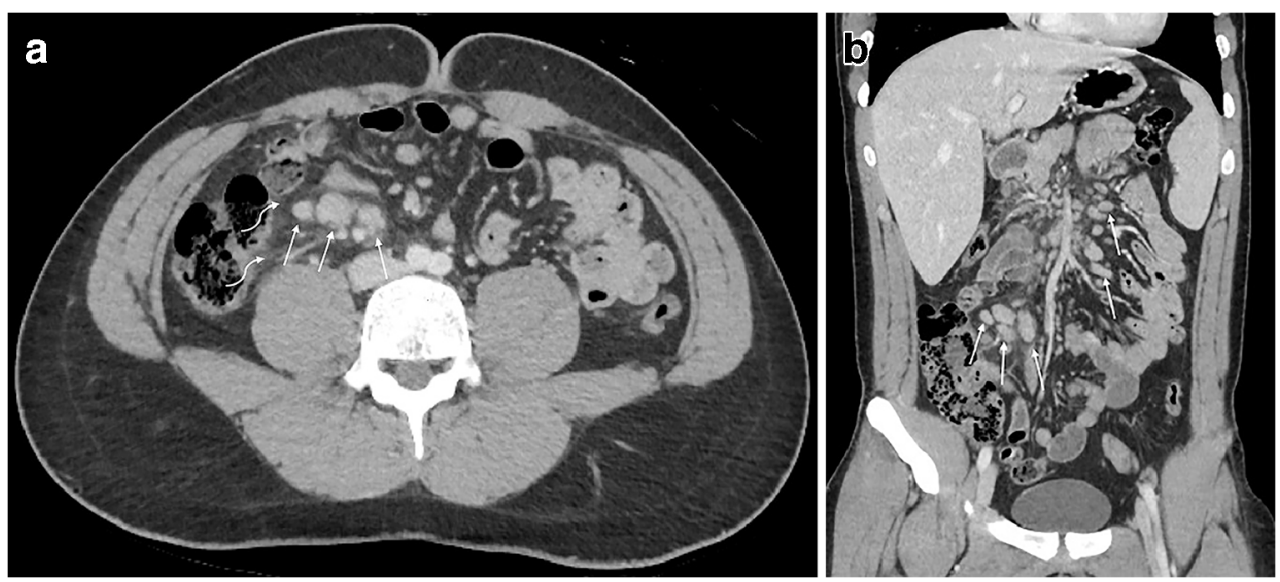
Table 1 Nasopharyngeal, blood and stool tests (all with negative results) performed in addition to cultures and COVID-19 testing

\begin{tabular}{lcc}
\hline Nasal swab PCR testing & Blood viral testing & Stool testing \\
\hline Multiplex respiratory PCR: & Adenovirus PCR: & Stool multiplex PCR: \\
Adenovirus & HIV antigen and antibody & Campylobacter (jejuni, coli, upsaliensis) \\
Coronavirus 229E & CMV IgG and IgM & Plesiomonas shigelloides \\
Coronavirus HKU1 & EBV PCR & Salmonella species \\
Coronavirus NL63 & EBV IgG, IgM, nuclear & Yersinia enterocolitica \\
Coronavirus OC43 & antibody, early & Enterotoxigenic E. coli (ETEC) \\
Human metapneumovirus & antigen antibody & E. coli O157 STEC \\
Human rhinovirus/ enterovirus & & E. coli NON O157 STEC \\
Influenza A & & Shigella species \\
Influenza A subtype H1 & & Vibrio (parahaemolyticus, \\
Influenza A subtype 2009 H1 & & vulnificus, cholerae) \\
Influenza A subtype H3 & Cryptosporidium \\
Influenza B & & Cyclospora cayetanensis \\
Parainfluenza virus 1 & & Entamoeba histolytica \\
Parainfluenza virus 2 & & Giardia duodenalis \\
Parainfluenza virus 3 & & Adenovirus F 40/41 \\
Parainfluenza virus 4 & & Astrovirus \\
Respiratory syncytial virus & & Norovirus \\
Mycoplasma pneumoniae & & Rotavirus \\
Bordetella pertussis & & Sapovirus \\
Chlamydophila pneumoniae & & \\
Methicillin-resistant Staphylococcus aureus PCR screen & & \\
\hline
\end{tabular}

$C M V$ cytomegalovirus, $E$. coli Escherichia coli, EBV Epstein-Barr virus, $H I V$ human immunodeficiency virus, $\operatorname{Ig} G$ immunoglobulin G, $P C R$ polymerase chain reaction, STEC Shiga toxin-producing E. coli

inflammatory bowel disease were excluded, and extensive viral and bacterial testing for other infectious causes was negative. There was no clinical or imaging evidence of bowel ischemia.

Symptomatic COVID-19 infection most commonly manifests with respiratory symptoms. CT of early disease typically demonstrates pulmonary ground-glass opacities or normal $\mathrm{CT}$, and the intermediate stage manifests with increased ground-glass opacities as well as multifocal consolidative opacities, septal thickening and crazy paving pattern of pulmonary opacity [4]. Our patient initially presented after 3 days of symptoms without pulmonary findings suggestive of COVID-19 but subsequently developed pulmonary symptoms and imaging findings characteristic of COVID-19.

COVID-19 also often presents with gastrointestinal symptoms including diarrhea, nausea, vomiting and abdominal pain [3]. Nucleic acid testing can detect SARS-CoV-2 in stool samples even in patients without diarrhea [5]. However, it is unknown whether viral particles in stool are infectious because no direct fecal-oral transmission has been reported.

Acute mesenteric adenopathy is typically a self-limiting inflammatory disease in the absence of identifiable causes such as acute appendicitis, inflammatory bowel disease, infectious mononucleosis, yersiniosis and salmonella infection and in the absence of complications such as ileocolic intussusception [6]. SARS-CoV-2 has been shown to infect and replicate within human enterocytes in vitro, which provides a putative mechanism for mesenteric adenopathy given the abundant lymphatic tissue in and associated with the bowel [7].

The most common laboratory methods for diagnosing COVID-19 are based on nucleic acid amplification or PCR from upper and lower respiratory tract, blood, stool and urine samples. These tests are very specific, but sensitivity can vary depending on sample type, severity of disease, and where the patient is in his or her disease course. In general, upper respiratory samples are more sensitive early in disease, with lower respiratory samples more sensitive later in disease [5]. In our patient's case, even though multiple earlier upper respiratory tract tests were negative, imaging and symptoms suggested progressive lower tract COVID-19, which highlights the need for continued or alternative testing if clinical suspicion remains high.

This case report describes a severe case of COVID-19 in a previously healthy teenage patient who initially presented with gastrointestinal symptoms and isolated acute mesenteric adenopathy on imaging. Screening for COVID-19 by two nasopharyngeal swabs was negative before a positive SARS-CoV-2 PCR from sputum confirmed the diagnosis. Our case report demonstrates that children with clinical findings highly suspicious for acute appendicitis might actually be manifesting an atypical presentation of COVID-19. A high index of suspicion should be maintained for COVID-19 in all patients given the continued emergence of new manifestations of the disease. 
Acknowledgments The authors express their gratitude to the patient and his family for permission to share this case.

\section{Compliance with ethical standards}

Conflicts of interest None

\section{References}

1. World Health Organization (2020) Coronavirus disease (COVID-19) situation report - 176. WHO website. https:/www.who.int/docs/ default-source/coronaviruse/situation-reports/20200714-covid-19sitrep-176.pdf?sfvrsn=d01ce263 2. Accessed 15 July 2020

2. Dane B, Brusca-Augello G, Kim D, Katz DS (2020) Unexpected findings of coronavirus disease (COVID-19) at the lung bases on abdominopelvic CT. AJR Am J Roentgenol. https://doi.org/10. 2214/AJR.20.23240

3. Bhayana R, Som A, Li MD et al (2020) Abdominal imaging findings in COVID-19: preliminary observations. Radiology. https://doi.org/ 10.1148/radiol.2020201908

4. Salehi S, Abedi A, Balakrishnan S, Gholamrezanezhad A (2020) Coronavirus disease 2019 (COVID-19): a systematic review of imaging findings in 919 patients. AJR Am J Roentgenol 215:87-93

5. Wang W, Xu Y, Gao R et al (2020) Detection of SARS-CoV-2 in different types of clinical specimens. JAMA 323:1843-1844

6. Helbling R, Conficconi E, Wyttenbach M et al (2017) Acute nonspecific mesenteric lymphadenitis: more than "no need for surgery". Biomed Res Int 2017:1-4

7. Lamers MM, Beumer J, van der Vaart J et al (2020) SARS-CoV-2 productively infects human gut enterocytes. Science 369:50-54

Publisher's note Springer Nature remains neutral with regard to jurisdictional claims in published maps and institutional affiliations. 